DOI: 10.20472/IAC.2018.039.044

\author{
GURKAN TUNA \\ Trakya University, Turkey \\ RESUL DAŞ \\ Fırat University, Turkey
}

\title{
MOBILE APPLICATION FOR DIABETES FOLLOW-UP AND ANALYSIS
}

\begin{abstract}
:
It is very difficult for people with diabetes, one of the most common diseases of recent years, to follow factors that affect their disease. With the development of technology, patients have become able to measure their own blood sugar using tiny devices whenever they want, wherever they want, instead of going to hospitals to measure blood sugar. Considering that nowadays when smartphones are so widespread, diabetes can be easily followed using a mobile application. In this paper, design and development of a mobile application that allows a patient to save his/her diabetes related parameters at certain intervals and transmit the values to his/her doctor is explained. Since potential users of the designed application are Turkish people, the interfaces of the software application are in Turkish. The user interface in English is currently being developed.
\end{abstract}

\section{Keywords:}

Diabetes, Follow-up, Analysis, Software, Mobile Application, Android.

JEL Classification: C88, L86 


\section{Introduction}

It is known that currently there are around 250 million diabetics (Tabish, 2007). Blood sugar follow-up is one of the most important steps in the treatment of diabetes. Regularly measured blood sugar helps to confirm diabetes control by providing a comparison with past values while providing information about glucose values (Aliha et al., 2013). Blood sugar values are used to adjust the way they are fed, as well as to see how the nutrients taken affect blood sugar. In this view, blood sugar can be controlled by applying individual diets (Gray, 2000).

It is very difficult for people with diabetes to follow factors that affect their daily glucose and diabetes. However, with the development of technology, patients have become able to measure their own blood sugar, using a small device whenever they want, wherever they want, instead of going to hospitals to measure blood sugar.

Since nowadays smartphones are widespread and the usage rates of smartphones are increasing very rapidly (Garden, 2016), it would be good and comfortable if diabetes can be controlled using mobile applications. In this study, a mobile application which allows the patient to take glucose values and / or carbohydrate, fast / short insulin and medium / long insulin values taken at certain intervals, transmit the relevant doctor online via mobile application and enable the doctor to give advice to the user is proposed. In addition, the user will be able to regularly share information about weight, ketone and blood pressure with his / her doctor and leave special notes for the doctor.

\section{Proposed Mobile Application}

The proposed application is supposed to work on the Android platform. Since diabetes may occur in people of all ages, the application should be designed to meet the needs of people of all ages and its graphical interface should be user friendly, simple and easily accessible. To be able to use the application, users must first create accounts for themselves. The information provided by the users will be stored securely considering the confidentiality of sensitive information. The data accumulated over time will be used by the doctors and allow the doctors to see the general condition of their patients over time. Also, the patients and doctors will be able to communicate through the message panel.

To run the developed application, Android 4.0.3 (API Level: 15) or higher is required. The application has the responsive feature provided by the Android library and preserves its visual design in all screen resolutions. Hence, there is no screen resolution limit. In order for the patient-doctor interaction to be carried out, which is the most important purpose of the application, the application requires internet connection.

Spiral model has been used as software development methodology. Since the application's target platform is the Android platform, Android Studio (https://developer.android.com/studio/) has been used as the development environment 
and JAVA programming language has been used for the development. In the server-side development, the Eclipse Neon development environment has been preferred due to its rich add-ons (http://www.eclipse.org/neon/). Tomcat Server 8.5 (http://tomcat.apache.org/) has been used for the Java server, and appropriate Java classes have been created to allow the server side to communicate with the Android application. Since communication between the server and the mobile application is fast compared to all other methods, it is provided by the REST method and RESTful web services are used (Hamad, Saad, and Abed, 2010). MySQL, an open source database management system, is used as the database management system. Text / HTML method is used for data transfer. As shown in Figure 1, the system has 3-tier architecture: User, Server and Database Server. The server part serves as a bridge between the user and the database server, and according to the request from the user, transmits the data in the database to the user application in JSON format and the application displays this data in visual designs by the user. Tomcat 8.5 Java library has been used as the system server and a Java server has been created. For the REST service, using the Jersey RESTful Web Services Framework, the server has been designed and JSON has been chosen as the data format. Information coming from the Android application to the server are added, deleted and edited in the required database tables, through JSON parsing. Overall operation of the proposed application is shown in Figure 2. The developed application's graphical user interfaces are shown in Figures 3-5.

\section{Figure 1: System architecture}
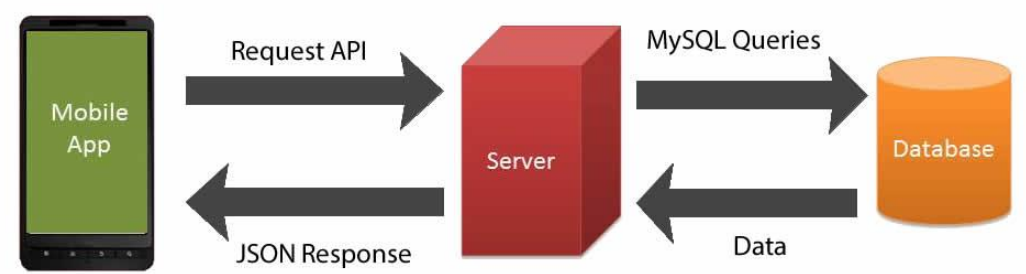
Figure 2: Overall operation of the proposed application

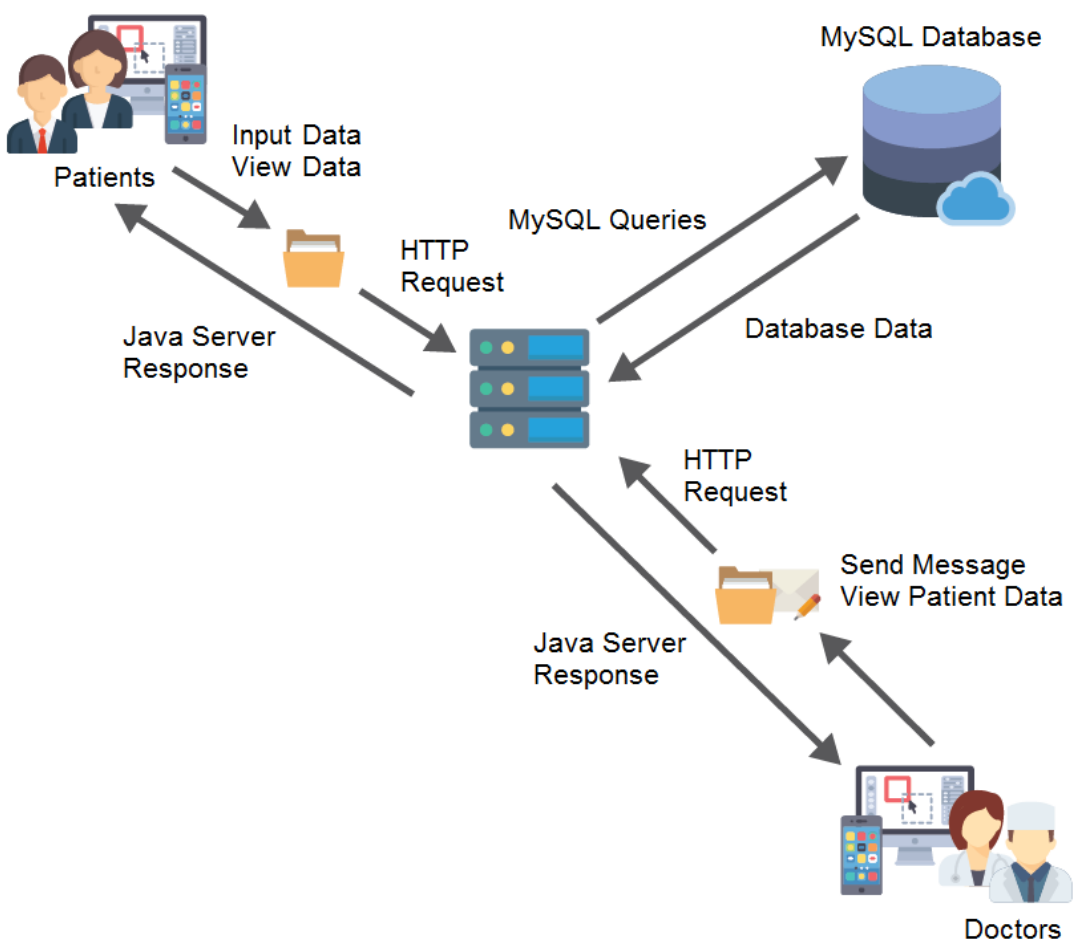

Figure 3: (a) User login interface of the mobile application, (b) Main interface of the mobile application

(a)

(b)

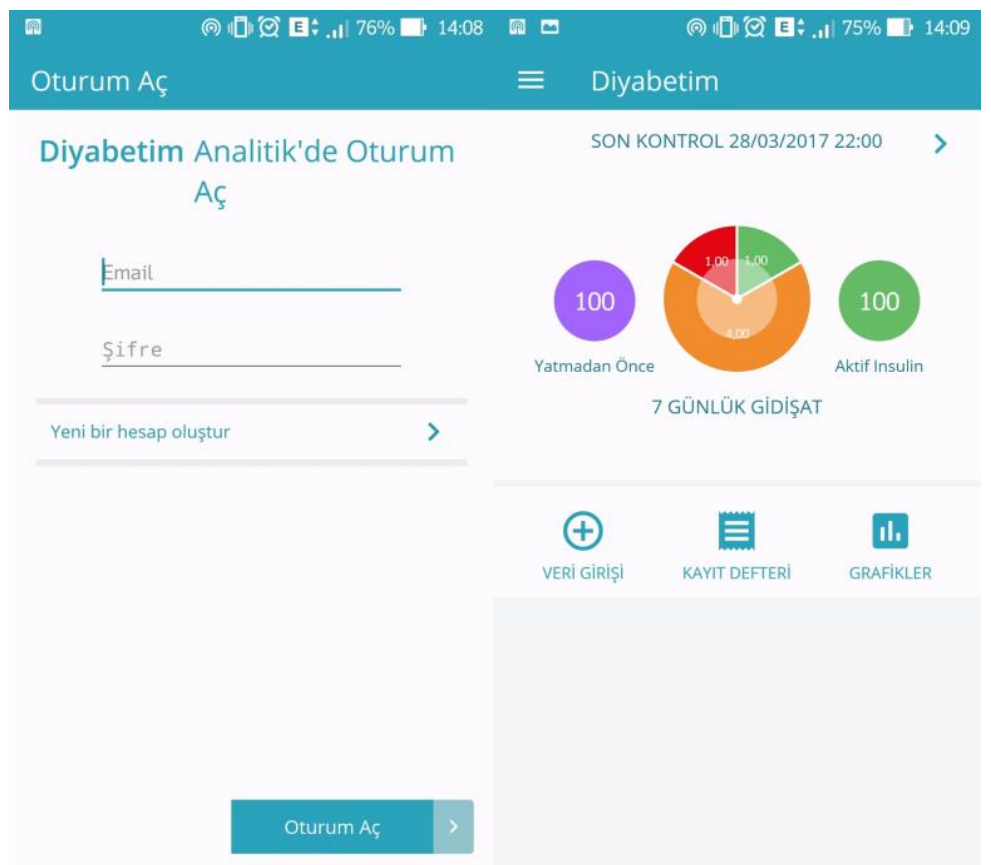


Figure 4: Inputting data using the interface

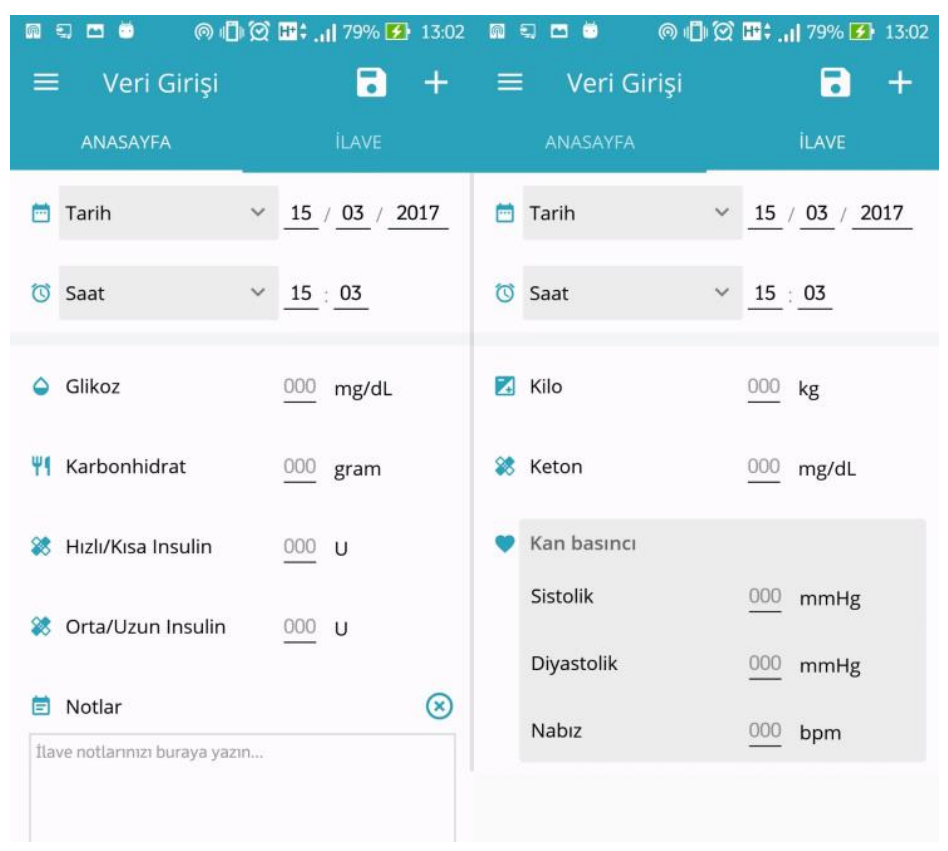

\section{Figure 5: Checking the history}

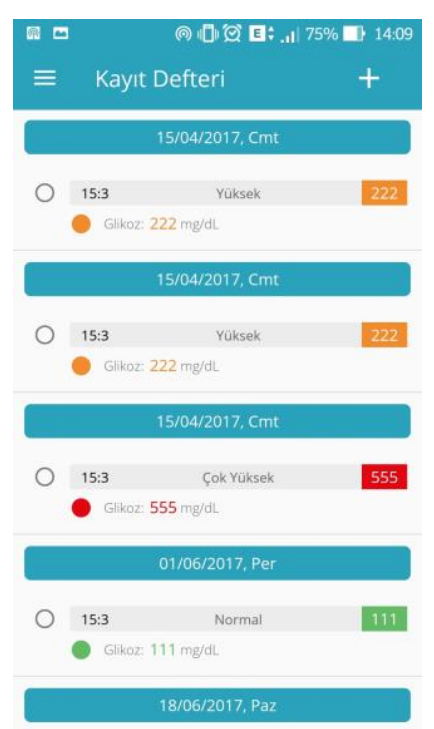

\section{Conclusion}

Thanks to the developed mobile application and its simple interface, by providing patientdoctor interaction, blood glucose and insulin intake of the application's users can easily be ensured and analysis can be done through graphs. Using their smartphones and the developed application, the users can control their diabetes and can communicate with 
their doctors whenever they want. This way, the doctors can closely monitor their patients' health and see the progress of the disease with graphic support.

\section{References}

Aliha, J. M., Asgari, M., Khayeri, F., Ramazani, M., Farajzadegan, Z., and Javaheri, J. (2013). Group Education and Nurse-Telephone Follow-Up Effects on Blood Glucose Control and Adherence to Treatment in Type 2 Diabetes Patients. International Journal of Preventive Medicine, 4(7), pp. 797802.

Garden, G. (2016). Psychiatric Care of the Medical Patient, 3rd edition. The British Journal of Psychiatry, 209(1), p. 87.

Gray, A. (2000). Nutritional Recommendations for Individuals with Diabetes, In L. J. De Groot, G. Chrousos, K. Dungan, et al. (Eds.), Endotext [Internet]. South Dartmouth (MA): MDText.com, Inc. Available from: https://www.ncbi.nlm.nih.gov/books/NBK279012/

Hamad, H., Saad, M., and Abed, R. (2010). Performance Evaluation of RESTful Web Services for Mobile Devices. International Arab Journal of e-Technology, 1(3), pp. 72-78.

Tabish, S. A. (2007). Is Diabetes Becoming the Biggest Epidemic of the Twenty-first Century?. International Journal of Health Sciences, 1(2), pp. V-VIII.

https://developer.android.com/studio/index.html

http://www.eclipse.org/neon/

http://tomcat.apache.org/ 